\title{
Energy Conservation for GPU-CPU Architectures with Dynamic Workload Division and Frequency Scaling ${ }^{\sqrt{2}}$
}

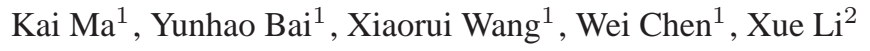 \\ ${ }^{1}$ Department of Electrical and Computer Engineering, The Ohio State University, USA \\ ${ }^{2}$ Department of Electrical Engineering and Computer Science, The University of Tennessee, Knoxville, USA
}

\begin{abstract}
In recent years, GPU-CPU heterogeneous architectures have been increasingly adopted in high performance computing, because of their capabilities of providing high computational throughput. However, the energy consumption is a major concern due to the large scale of such kind of systems. There are a few existing efforts that try to lower the energy consumption of GPU-CPU architectures, but they address either GPU or CPU in an isolated manner and thus cannot achieve maximized energy savings. In this paper, we propose GreenGPU, a holistic energy management framework for GPU-CPU heterogeneous architectures. Our solution features a two-tier design. In the first tier, GreenGPU dynamically splits and distributes workloads to GPU and CPU based on the workload characteristics, such that both sides can finish approximately at the same time. We comparatively discuss four dynamic workload allocation algorithms: a simple heuristic with fixed step size, an improved heuristic with adaptive step size, and two binary search-style algorithms. As a result, the energy wasted on idling and waiting for the slower side to finish is minimized. In the second tier, GreenGPU dynamically throttles the frequencies of GPU cores and memory in a coordinated manner, based on their utilizations, for maximized energy savings with only marginal performance degradation. Likewise, the frequency and voltage of the CPU are scaled similarly. We implement GreenGPU using the CUDA framework on two real hardware testbeds. Experiment results show that GreenGPU achieves $21.04 \%$ average energy savings and outperforms several well-designed baselines.
\end{abstract}

Keywords: GPU, energy efficiency, workload division, dynamic frequency scaling.

\section{Introduction}

In recent years, GPU-CPU heterogeneous architectures have been increasingly adopted in high performance computing, because of their capabilities of providing high computational throughput. For example, one of the world's fastest supercomputers, Titan [28], is equipped with AMD Opteron 6274 processors and Nvidia's CUDA-enabled NVIDIA K20x general purpose GPUs. GPUs excel at data parallel operations due to the optimized SIMD (Single Instruction Multiple Data) structure. Given the same amount of data, using one instruction to process multiple pieces of data can be more efficient than one instruction for one piece of data in both performance and energy. This advantage has helped Titan to achieve an impressive 2142.8MFLOP/W power efficiency [28]. However, the

\footnotetext{
ॠThis is a significantly extended version of a conference paper [22]. This work was supported, in part, by NSF under grants CNS-1143605, CNS0915959, and CNS-1143607 (NSF CAREER Award), and by ONR under grant N00014-09-1-0750.

Email addresses: ma.495@osu. edu (Kai Ma ${ }^{1}$ ), bai.228@osu.edu (Yunhao Bai ${ }^{1}$ ), wang. 3596@osu.edu (Xiaorui Wang ${ }^{1}$ ), chen.2890@osu.edu (Wei Chen ${ }^{1}$ ), xli44@utk.edu (Xue $\left.\mathrm{Li}^{2}\right)$
}

Preprint submitted to Elsevier total power consumption of Titan system also hits $8209 \mathrm{~kW}$, an estimated annual electricity bill of more than $\$ 4$ million [8]. Therefore, it is important to further improve the energy efficiency of GPU-CPU heterogeneous architectures.

GPU-CPU heterogeneous architectures offer some unique opportunities for energy conservation. Since GPUs have energy efficiency advantage over CPUs for parallel and computationintensive applications, an intuitive solution seems to be assigning all those workloads to the GPU for energy efficiency. However, our experiments (in Section 3.2) show that the GPU taking all the workloads is not necessarily the most energyefficient workload division. The main reason is that if the GPU takes all the workloads while the CPU is totally idling, the execution time of the entire system can be longer than that in the case when the CPU does a fair portion of work. Since energy is the product of time and power, a more energy-efficient solution is to split and distribute the workload to GPU and CPU, such that both sides can finish approximately at the same time. However, because CPUs and GPUs differ considerably in their processing and memory capabilities, it is challenging to design an algorithm that can achieve an energy-efficient workload division for all different workloads. Furthermore, dif- 
ferent power adaptation knobs, such as frequency (and voltage) scaling, are commonly enabled in both CPUs and GPUs. Since frequency scaling may impact the hardware capabilities, workload division policies assuming fixed underlying hardware working status might lead to inferior workload allocation. Therefore, a dynamic workload division algorithm aware of the hardware status needs to be designed.

After the workload is split and allocated to the GPU and $\mathrm{CPU}$, another research challenge is to manage hardware resources according to the runtime needs of workloads for energy savings without compromising performance. In GPUs, real-world applications rarely fully stress the GPU cores and memory simultaneously [12]. Hence, there are opportunities to save energy by throttling the components with low utilizations. For example, for GPU core-bounded workloads, we can throttle the memory frequency for energy savings. However, a naive solution may over-throttle the memory and thus make the memory part become the system bottleneck, resulting in unnecessary performance degradation. A similar argument also applies to memory-bounded workloads. Therefore, the GPU cores and memory must be managed in a coordinated manner, based on the workload characteristics, for energy savings with minimal performance degradation. However, existing research on GPU energy management focuses on either GPU cores or memory. The direction of coordinately throttling the frequency levels of both the GPU cores and memory for improved GPU energy efficiency needs to be investigated.

In this paper, we propose GreenGPU, a holistic energy management framework for GPU-CPU heterogeneous architectures. Our solution features a two-tier design. In the first tier, GreenGPU dynamically splits and distributes workloads to GPU and CPU based on the workload characteristics, such that both sides can finish approximately at the same time. We comparatively discuss four dynamic workload allocation algorithms: a simple heuristic with fixed step size, an improved heuristic with adaptive step size, and two binary search-style algorithms. As a result of dynamic workload allocation, the energy wasted on idling and waiting for the slower side is minimized. In the second tier, GreenGPU dynamically throttles the frequencies of the GPU cores and memory in a coordinated manner, based on their utilizations, for maximized energy savings with only minimal performance degradation. Likewise, the frequency and voltage of the CPU are scaled similarly. Specifically, this paper makes following contributions:

- We propose to improve the energy efficiency of GPUCPU heterogeneous architectures in a holistic way to utilize both workload division and frequency scaling for maximized energy savings.

- We design a two-tier solution that dynamically splits and distributes workloads to GPU and CPU in the first tier and throttles the frequencies of GPU cores, GPU memory, and CPU cores in the second tier.
- We develop a light-weight machine learning algorithm to adjust the frequency levels for the GPU cores and memory in a coordinated manner, based on the workload characteristics, for energy conservation.

- We discuss and compare four workload distribution algorithms to dynamically assign the workload on both CPU and GPU for minimized idling power waste on both sides.

- We implement GreenGPU using the CUDA framework on a real physical testbed with Nvidia GeForce GPUs and AMD Phenom II CPUs. Experiment results with standard Rodinia benchmarks show that the proposed GreenGPU framework achieves $21.04 \%$ average energy savings than several well-designed baselines.

The rest of the paper is organized as follows. Section 2 highlights the difference between this paper and related work. Section 3 motivates our work with two case studies. Section 4 presents our overall system design at a high level. Section 5 introduces in detail the algorithms proposed for dynamic frequency scaling and workload division. Section 6 provides the implementation details of our testbed, while Section 7 discusses our hardware experimental results. Finally, Section 8 concludes this paper.

\section{Background}

Recently, workload division between CPU and GPU has drawn the attention of researchers. Luk et al. [21] propose Qilin framework, an adaptive mapping scheme to map computation tasks to processing elements on the CPU and GPU in one machine. While their target is solely to minimize the execution time, our scheme integrates workload division with GPU core and memory throttling to improve the energy efficiency of the entire system. Wang et al. [32] propose to coordinate inter-processor work distribution to minimize energy consumption under a given scheduling length constraint. However, their work does not throttle the GPU memory and cores in a coordinated manner based on workload characteristics for maximized energy savings. In addition, their approach requires offline profiling, which may be undesirable because it can be expensive to do profiling for applications with a large amount of data every time for different input variables. Some GPU-CPU workload division studies are conducted based on the MapReduce [4] framework. For example, Ravi [30] proposes dynamic input data partitioning among CPU cores and GPU cores based on two applications, $K$-means clustering and Principal Component Analysis. Hong et al. [11] discuss a uniform memory management framework among CPU and GPU as well as a uniform programming API. While both the studies aim at improving the code portability between CPU and GPU, GreenGPU addresses a different problem, i.e., improving the energy efficiency of GPU-CPU heterogeneous architectures. 

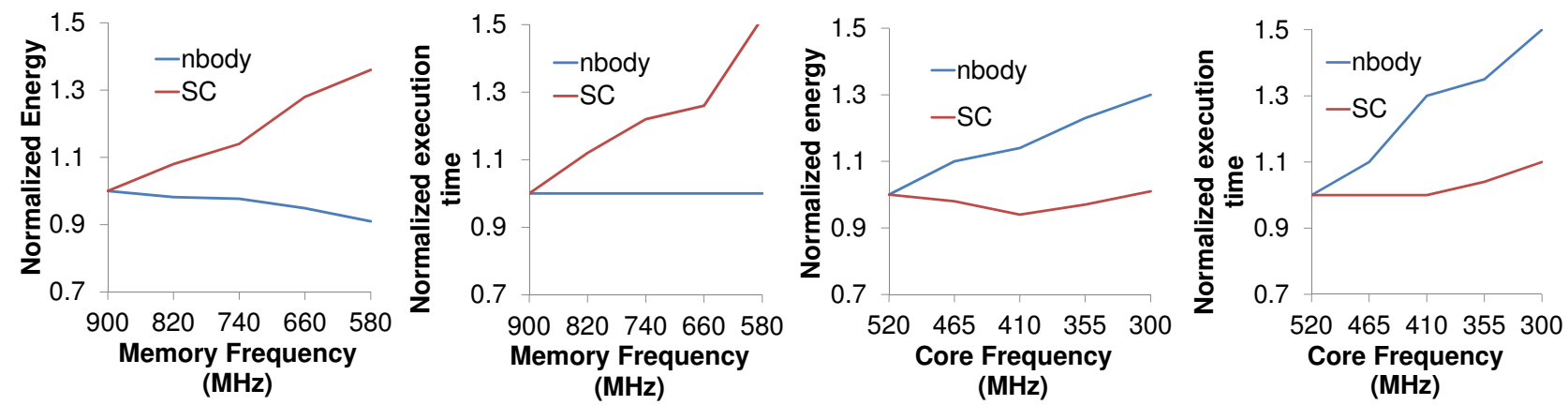

(a) Impact of memory frequency scal-(b) Impact of memory frequency scal-(c) Impact of core frequency scaling(d) Impact of core frequency scaling ing on energy ing on performance on energy

on performance

Figure 1: Normalized execution time is the execution time of a workload normalized to its execution time at the peak frequency. Relative energy is the energy normalized to the energy consumed at the peak frequency. There are opportunities to save energy with negligible performance loss by throttling under-utilized components.

There are some existing research efforts to improve the energy efficiency of GPUs but those studies address GPU cores and memory in an isolated manner. Hong et al. [12] have shown that throttling the number of GPU cores based on their novel power model can save energy. Based on their power measurement, Collange et al. [3] conclude that memory access pattern and bandwidth play a major role in achieving both good performance and low power consumption. Jang et al. [15] formulate the memory access pattern of the threads inside a computation kernel in a matrix to select runtime parameters. Compared with those previous studies that address either GPU cores or memory, GreenGPU coordinates both GPU cores and memory for maximized energy savings. Recently, Lee et al. [17] have studied to coordinate the number of active cores, the frequency of core part, and cache part in GPUs. However, the coordination between CPU and GPU (one of the key components in GreenGPU) is not examined in their work.

The general energy saving techniques of CPUs have been researched extensively $[13,34,10,18,1]$. Particularly, some projects have tried to improve the energy efficiency of chip multiprocessors running parallel applications. For example, $\mathrm{Li}$ et al. [18] propose a solution called thrifty barrier that places the faster cores into a lower power mode at the barriers (i.e., joint point) while waiting for the slower cores so that energy can be saved. Liu et al. [20] use per-core DVFS to slow down the faster cores, such that both the idle time due to waiting and energy consumption are reduced. Cai et al. [1] extend [20] by adding meeting points within the execution of the parallel loops and solve the same problem at a finer granularity. However, all these studies focus on CPU-only architectures without considering GPU as part of the system, so their methods could not be directly applied to the GPU-CPU heterogeneous architectures.

\section{Motivation}

In this section, we conduct two case studies: 1) frequency scaling on GPU cores and memory, and 2) workload division between CPU and GPU, to motivate our work.

\subsection{A Case Study on Frequency Scaling for GPU Cores and Memory}

In GPUs, real-world applications rarely stress both the core and memory parts at the same time [12]. The component (e.g., cores and memory) utilization measures how intense one workload is exercising one part of the hardware resource. Nvidia defines the core part utilization as "GPU busy cycles/total cycles" and memory utilization as "actual bandwidth/rated peak bandwidth" [25]. We get the utilizations by using Nvidia's nvidia-smi toolset. Based on the utilization trace analysis, we categorize nbody as core-bounded and streamcluster (SC) as memory-bounded. nbody and SC are workloads in CUDA SDK. In the following experiments, we conduct experiments on Nvidia GeForce 8800 GTX GPU to explore energy saving opportunities.

Figures 1a and 1b illustrate the energy and performance when we throttle the memory frequency. The frequency of the cores is set to the peak value. The opposite case is presented in Figures 1c and 1d. In Figures 1a and 1b, reducing the frequency of the memory part saves energy with minor performance loss for core-bounded nbody; but it impacts both performance and energy for memory-bounded $S C$. Figures 1c and $1 \mathrm{~d}$ show that reducing the frequency of core part negatively impacts both performance and energy for core-bounded nbody. For $S C$, reducing the frequency of core part to certain point (e.g., $410 \mathrm{MHz}$ ) saves energy with negligible performance loss; further reducing the frequency of core part negatively impacts both performance and energy. We make the following two observations based on the experiments: 


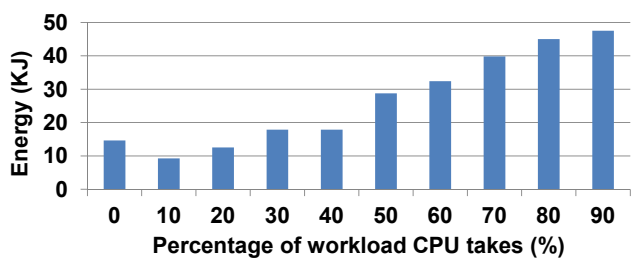

Figure 2: Energy consumption for different workload division ratios. The cooperation of the CPU and GPU parts can be more energy efficient than the GPU part taking all the work exclusively.

1. For either memory-bounded or core-bounded workloads, properly scaling down the under-utilized component can save energy with negligible performance impact.

2. To either CPU or GPU at a certain utilization level, there can be a frequency level of that component that is most suitable, i.e., a higher frequency may lead to higher energy consumption while a lower frequency may result in performance loss.

In this paper, we aim to design frequency scaling algorithms that dynamically adjust the frequency levels according to the measured utilizations of the cores and memory.

\subsection{A Case Study on Workload Division between GPU and $C P U$}

Although GPUs have theoretical energy-efficiency in parallel computing, CPUs may participate in the computation to provide even higher throughput for the whole system. Luk et al. [21] give a workload division case study to show that different workload divisions between the CPU and GPU parts yield different performance. In Figure 2, we conduct similar experiments to investigate the relation between energy consumption and workload division. Implementation details are introduced at Section 6. We measure the energy consumption of the entire GPU-CPU system when we vary the percentage of work allocated to CPU from $0 \%$ to $90 \%$. The example case is based on the kmeans workload from the Rodinia benchmark set [2]. We observe that the energy consumption goes down as CPU work percentage goes from $0 \%$ to $10 \%$, and then goes up from $10 \%$ to $90 \%$. The optimal point takes place when CPU side takes $10 \%$ of the total work. Figure 2 shows that the cooperation of the CPU and GPU parts can be more energy efficient than the GPU part taking all the work exclusively. On a GPU-CPU heterogeneous platform, the average energy/data efficiencies (i.e., the joules consumed per certain amount of data [9]) on the GPU and CPU sides are different. Given a fixed amount of workload (fixed amount of data), the energy optimization problem can be abstracted as: Given a GPU-CPU system with a GPU and a CPU, a certain amount of workload as $x$, the energy coefficient of the CPU and GPU as $a_{1}$ and $a_{2}$, respectively, we need to find a workload division $x_{1}$ for GPU and $x_{2}$ for CPU and $x_{1}+x_{2}=x$, such that $a_{1} * x_{1}+a_{2} * x_{2}$ is minimized. This optimization problem is not trivial because $a_{1}$ and $a_{2}$ are different for different workloads and may change over time. So the minimum point may also change over time. In this paper, we aim to design a workload division algorithm that dynamically adjusts the workload division to find the energy minimized point at runtime.

\section{System Design of GreenGPU}

In this section, we first introduce a typical hardware configuration of GPU-CPU heterogeneous architectures. We then present the two-tier design of GreenGPU, targeting at reducing the system energy consumption. Finally, we discuss the interaction between the two tiers in GreenGPU.

The lower part of Figure 3 shows the logic view of a typical GPU-CPU heterogeneous platform. The CPU, main memory, and the GPU are connected by the system bus. CPU works as the master and GPU works as the slave in this configuration. Although GPU is a slave device, it has DMA (Direct Memory Access) to improve memory capability. The CPU and GPU parts have very different architectures. Compared with CPU, the GPU part is equipped with enhanced stream processors (SP) organized as stream multiprocessors (SM) to perform high throughput computation. The SIMD architecture of GPUs is highly optimized for data parallelism and has higher energy efficiency than CPUs for certain parallel workloads.

As shown in the upper part of Figure 3, GreenGPU is a two-tier solution, running on the CPU. GreenGPU includes a workload division unit and two frequency scaling units (one for CPU and one for GPU, respectively). Both the workload division and the frequency scaling are invoked periodically, however, with different invocation periods.

In the first tier, the workload division unit dynamically divides the workloads between CPU and GPU based on their execution time to reduce the idling energy. The dynamic workload division takes place at every iteration. An iteration is defined as the execution of a fixed amount of work. The iteration selection is workload dependent. An iteration could be the execution to the reduction point in the algorithm of a workload (e.g., the iteration in kmeans). Or it could be the execution to the common barrier point in a workload with barriers (e.g., the step in hotspot). Since the operations inside each iteration are similar [24], the statistics collected during the previous iteration can serve as a prediction for the execution of the next iteration. Our target is to dynamically adjust the workload division in every iteration to minimize the execution time difference between the GPU and CPU parts for minimized idle energy. If in the previous iteration, the CPU runs slower than the GPU, we assign less work to the CPU and more work to the GPU in the next iteration and vice versa. Likewise, if in the previous iteration, CPU runs faster than GPU, we assign more work to the CPU and less to the GPU in the next iteration. This light-weight heuristic reduces the idling time by workload division between the CPU and GPU parts to reduce idle energy. 


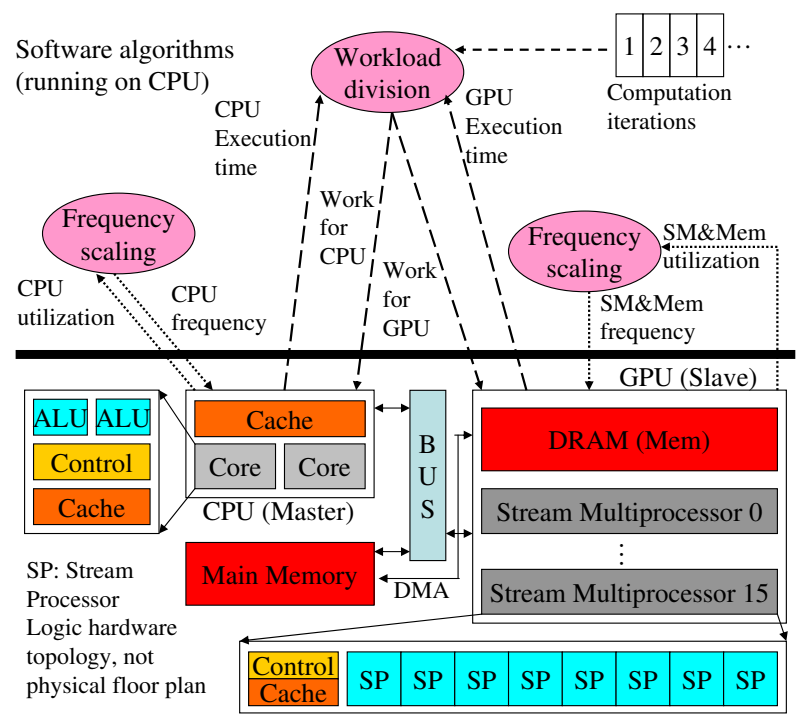

Figure 3: GreenGPU features a two-tier design to reduce the energy consumption of CPU-GPU heterogeneous platforms. The higher tier (i.e., the workload division tier) dynamically partitions the incoming workloads to the CPU and GPU parts. The dash lines connect the components of the workload division part. The lower tier (i.e., the frequency scaling tier) takes the utilization of processing elements (GPU cores, GPU memory, and CPUs) to decide the proper frequency levels of them to reduce energy consumption. The dotted lines connect the components of the frequency scaling part.

Please note although we give two examples for iterations, the iteration selection is not limited to these two types. As long as the next iteration execution time on the CPU and GPU parts can be reasonably predicted based on current iteration, those iteration selections are valid. For example, for workloads that all the working threads are totally independent, the whole data set could be divided into a series of chunks. We refer to each run on a chunk as an iteration.

In the second tier, once the workload is divided and assigned to the CPU and GPU, the two frequency scaling units in GreenGPU monitor the utilization of each component, and dynamically adjust the frequency levels of each component to achieve an improved energy efficiency. As we analyzed in detail in Section 3, component utilization metric can capture how intense the application is exercising the hardware. Highly utilized resource needs to be running at a high frequency level; low utilized resource can be throttled to save energy without significantly impacting performance. Since the GPU cores and memory interact with each other, we propose a coordinated algorithm to assign frequencies to the GPU cores and memory. The inputs of the algorithm are the utilizations of the GPU cores and memory in the current interval. The outputs of the algorithm are the target frequency levels of GPU cores and memory for the next interval. The detailed algorithm is presented in Section 5. For the CPU part, because there already exists rich research on dynamic voltage and frequency scaling (DVFS), we simply adopt the default Linux power saving strategy by setting CPU frequency policy mode to on-demand [29]. Ondemand is a dynamic kernel cpufreq governor that can change the CPU frequency depending on the CPU utilization. The ondemand governor works as follows. If CPU utilization rises above an upper utilization threshold value, the ondemand governor increases the CPU frequency to the highest available frequency. When CPU utilization falls below a low utilization threshold, the governor sets the CPU to run at the next lowest frequency. Ondemand was first introduced in the linux-2.6.9 kernel. It has been commonly used in a variety of systems with proven success. Please note that other more sophisticated DVFS-based processor power management strategies, such as $[13,34,31]$, can also be integrated into GreenGPU for even more energy savings. Similarly, memory DVFS on the CPU side [5] can also be integrated into GreenGPU for even more energy savings, which is our future work.

The workload division tier is invoked periodically to change the workload division between the CPU and GPU parts, which impacts the utilizations of the GPU cores, GPU memory and CPU. Therefore, to minimize the impact on the stability of the frequency scaling loops in the second tier, the period (i.e., iteration length) of the workload division tier is configured to be much longer than the period of frequency scaling to decouple the two loops. This design choice provides more flexibility for the design of each individual part. Alternatively, we could explore coupled algorithms. However, workload division commonly has higher overheads than frequency scaling and thus cannot be performed too frequently to deal with short-term workload variations. On the other hand, frequency scaling can be conducted more frequently due to its lower overheads. As a result, the two actuations are designed to run in different intervals for the trade-off between overhead and system responsiveness. For the GPU in our testbed, both the core and memory parts have 6 frequency levels. Therefore, the GPU frequency scaling loop may need 36 periods to test all the $36(6 \times 6)$ combinations of core and memory frequencies in the worst case. We select the workload division interval long enough (e.g., no less than 40 times longer than that of GPU frequency scaling interval) for the DVFS algorithm to converge to the optimal setting within one workload division interval. Therefore, before the next division starts, DVFS setting has already settled at the optimal point with the current division setting. Since the convergence time is normally short, the impacts of DVFS on workload division are observed to be negligible. Therefore, the two tiers will not interfere in a destructive way.

\section{GreenGPU Algorithms}

In this section, we first present our frequency scaling algorithm for GPU cores and memory. We then introduce our workload division algorithm. 


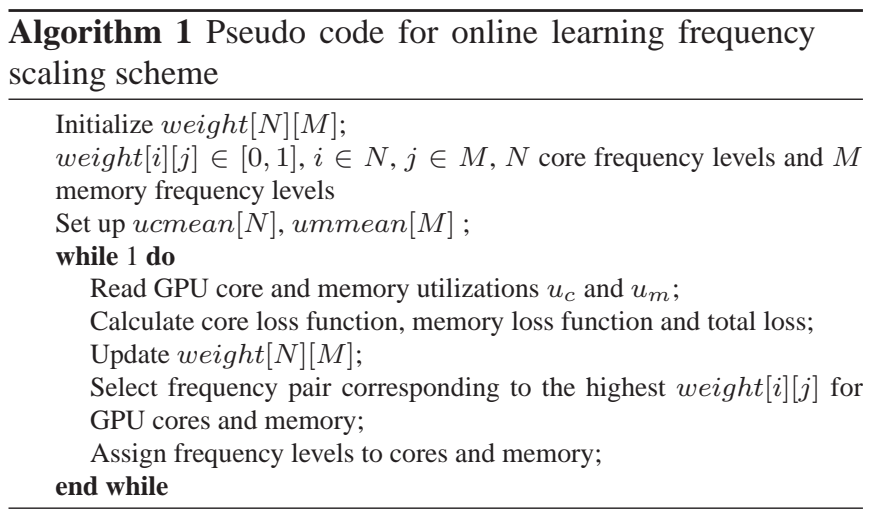

\subsection{Dynamic Frequency Scaling for GPU Cores and Memory}

Our dynamic frequency scaling algorithm aims at assigning frequency levels to GPU cores and memory to save energy with minimal performance loss. Since the GPU cores and memory parts interact with each other, we develop a coordinated algorithm to address this issue. We adopt the design framework of WMA (Weighted Majority Algorithm) [19] to develop our algorithm. In machine learning, WMA is a metalearning algorithm used to construct a compound algorithm from a pool of configurations. A weighted voting method could be used to find the optimal one(s) [7]. Specifically, we maintain a core-memory frequency pair weight table. Each field records the weight of one core-memory frequency pair. Those weights are updated according to the utilizations of the GPU cores and memory in the previous interval. The algorithm selects the core-memory pair with the highest weight to enforce in the next interval. Algorithm 1 explains the flow of our algorithm. We first initialize all of fields to an equal value (e.g., 0) since we do not have preference on any specific frequency level in the initial state. After the initialization, we periodically read the utilizations of GPU cores and memory, update the weight in each field based on its corresponding utilizations, then select the highest weight in the table and enforce the corresponding core-memory frequency pair. We can see the key part is how to update the weight, which we introduce in detail in the following paragraph.

As discussed in Section 3.1, highly utilized resource needs to run at a high frequency level while low utilized resource can be throttled to save energy without significantly impacting system performance. Therefore, for each component utilization, there may be a corresponding optimal frequency level. However, the available frequency levels in our system are discrete. Therefore, the purpose of our core-memory pair table is to evaluate how close the current utilization value is to the most suitable utilization of each core-memory frequency pair. The suitability for the current workload is represented by a loss factor $\left(0 \leq l_{i}^{t} \leq 1,1 \leq i \leq N, N\right.$ the number of available core levels, $t$ is the time interval index), which can be evaluated by comparing current utilization of the workload $u$ to the most suitable utilization of each available frequency
Table 1: Loss function used in the GPU frequency scaling algorithm

\begin{tabular}{|l|l|l|}
\hline Value of $u$ & Energy Loss $\left(l_{i e}^{t}\right)$ & Performance Loss $\left(l_{i p}^{t}\right)$ \\
\hline$u>$ umean $[i]$ & 0 & $(u-$ umean $[i])$ \\
\hline$u<$ umean $[i]$ & $($ umean $[i]-u)$ & 0 \\
\hline \multicolumn{2}{|l|}{$\operatorname{Loss}\left(l_{i}^{t}\right)=\alpha \times l_{i e}^{t}+(1-\alpha) \times l_{i p}^{t}$} \\
\hline
\end{tabular}

level umean. We define umean in the same way as in [6], which has been studied and validated on CPUs. We assume the peak frequency is suitable for utilization $100 \%$. The lowest frequency is suitable for utilization $0 \%$. And the other utilization and suitable frequency pairs are linearly mapped. Table 1 shows how to calculate the overall loss $\left(l_{i}^{t}\right)$. If current $u$ is smaller than the umean of a utilization level, then the workload stresses the resource less than the current frequency level can deliver. Hence, the resource can afford to run slower. This configuration has no performance loss $\left(l_{i p}^{t}\right)$, but since it could have saved energy by running slower, it has energy loss $\left(l_{i e}^{t}\right)$. Similarly, there is a performance loss, but no energy loss when $u>$ umean. Our metric includes both performance and energy to be general, because it offers a tradeoff between performance and energy. Although energy is the product of execution time and power, sometimes a DVFS setting with very low power consumption but a long execution time can be selected if its energy is the lowest. We include the trade-off to prevent this situation, because performance is the primary concern for many HPC applications. Our target is to save energy with only negligible performance degradation. $\alpha$ in Table 1 is a user-defined parameter that determines the relative importance of performance vs. energy savings. A smaller $\alpha$ directs the algorithm to favor performance while a larger $\alpha$ directs the algorithm to favor energy saving. In our system, since energy increases when performance degrades (i.e., a longer execution time), we give a higher weight to performance by setting $\left(\alpha_{c}=0.15\right.$ for cores and $\alpha_{m}=0.02$ for memory, $\alpha_{c}, \alpha_{m}$ are derived from experiments). Specifically, the loss factors for GPU cores and memory are calculated as:

$$
\begin{gathered}
l_{-} c_{i}^{t}=\alpha_{c} \times l_{-} c_{i e}^{t}+\left(1-\alpha_{c}\right) \times l_{-} c_{i p}^{t} \\
l_{\_} m_{j}^{t}=\alpha_{m} \times l_{-} m_{j e}^{t}+\left(1-\alpha_{m}\right) \times l_{-} m_{j p}^{t}
\end{gathered}
$$

Then we combine core and memory loss together by a factor $\phi$ to get the total loss in Equation 3. $\phi$ balances the impact of cores and memory on the system performance and energy saving. In our hardware testbed, $\phi=0.3$ is the value that reflects the system characteristic derived from experiments. $\phi$ is selected from experiments.

$$
\text { TotalLoss } s_{i j}^{t}=\phi \times l_{-} c_{i}^{t}+(1-\phi) \times l_{-} m_{j}^{t}
$$

Based on the total loss, the weights used in the frequency scaling algorithm can be updated as follows.

$$
\text { weight }_{i j}^{(t+1)}=\text { weight }_{i j}^{(t)}+\left(1-(1-\beta) \times \text { TotalLoss }_{i j}^{t}\right)
$$

In Equation 4, $\beta(0<\beta<1)$ is introduced to get the tradeoff between the current loss factor and the previous history 
weight. We select $\beta=0.2$ from experiments to filter out limited system noise with quick workload change response. Among the $N \times M$ weights (suppose we have $N$ core frequency levels and $M$ memory frequency levels), the highest one is selected and and its corresponding core and memory frequencies are enforced in the next period. Please note currently we derive $\alpha, \beta$, and $\phi$ from manual tuning due to the lack of accurate, general, and scalable performance/performance model for GPUs, which could be our future direction.

\subsection{Workload Division}

We now introduce how we use execution time as an indicator to divide workloads between the CPU and GPU parts. We comparatively discuss four dynamic workload allocation algorithms: a simple heuristic with fixed step size, an improved heuristic with adaptive step size, and two binary search-style algorithms.

Simple Heuristic. We define the percentage of work that CPU takes in an iteration as $r$, then GPU takes the rest $1-r$ percentage of the work. The time CPU uses to finish its work in an iteration is defined as $t_{c}$, while GPU's execution time is defined as $t_{g}$. Please note that $t_{g}$ and $t_{c}$ include data transfer time between main memory to GPU and CPU, respectively. When the system finishes the computation of the current iteration, the workload division unit will compare $t_{c}$ and $t_{g}$. If $t_{c}$ is longer than $t_{g}, r$ will be reduced by one step (e.g., one fixed amount, $5 \%$ ). If $t_{c}$ is shorter than $t_{g}, r$ will be increased by one step. The division step size can be decided by experiments. We test different step sizes in our experiments, but we use 5\% here for example. If we use a small step, the system takes a long time to converge to the optimal division point. There will be large oscillation if we use a large step size. Since the workload division is not continuous, there may be oscillation between two ratios. For example, with a 5\% step size, if the optimal division is $12.5 / 87.5$ (CPU/GPU), the system will oscillate between 10/90 (CPU/GPU) and 15/85 (CPU/GPU). In our experiments, this oscillation significantly degrades system performance due to the overheads of frequent workload division.

Therefore, we introduce a safeguard scheme to avoid this situation. Specifically, we linearly scale the execution times of the GPU and CPU in the previous iteration on both sides based on the possible workload allocation to predict the execution times in the next iteration. If the predicted execution times show that there can be oscillation, we keep using the current division for the next interval. For example, if we have $t_{c}<t_{g}$ for a division of 10/90 (CPU/GPU) in one iteration, we should take a fixed amount (e.g., 5\%) workload away from the GPU and give it to the CPU based on the algorithm. We now predict the execution times of GPU and CPU in the next iteration as $t_{c}^{\prime}=(15 / 10) * t_{c}$ and $t_{g}^{\prime}=(85 / 90) * t_{g}$, respectively. If $t_{c}^{\prime}>t_{g}^{\prime}$, oscillation may happen and so we keep the current division for the next interval. The above simple heuristic is straightforward in concept and easy to implement.
Those are desirable to serve as the starting point of validating the dynamic workload allocation idea. Note that our workload partitioning algorithms, which minimizes the waiting time of both the CPU and GPU side without any assumption on specific data sharing architectures, can also be directly applied to some new on-chip heterogeneous architectures (e.g., AMD APU, in which CPU and GPU share Last Level Cache).

Improved Heuristic with Adaptive Step Size. As discussed earlier, it can be difficult for the simple heuristic algorithm to find the best step size, because it depends on both the hardware platform and the workload. A small step size can lead to a long convergence time, while a large step size can result in a large distance from the optimal division point (with the safeguard scheme). Therefore, a key observation is that we can first use a large step size for faster convergence and then a smaller step size once oscillation occurs. Specifically, the algorithm starts with a large step size (e.g., 15\% or 20\%) and performs just like the previous heuristic with fixed step size. When the allocation starts to oscillate, it indicates that the optimal point is in the between of the previous two allocations and cannot be precisely reached due to the large step size. Then the algorithms halves the step size (e.g., to $7 \%$ or $10 \%$ ) and repeats the search process with the halved step size, such that we can reach closer to the optimal point. This process is repeated until the step size falls below a threshold (e.g, $1 \%)$. Compared to the heuristic with fixed step size, this improved heuristic can converge faster with an initial large step size and reach closer to the optimal point later with a small step size.

Binary Search Algorithm. As discussed before, the simple heuristic (with fixed step size) works well when it runs at coarse grain. For example, to explore $r$ (the dynamic range is $0 \%-100 \%$ ), if we take $20 \%$ as the workload allocation step, it will take 5 steps to explore the entire dynamic range. However, when we use fine allocation step size (e.g., 1\%), in the worst case, it takes 100 steps to converge to the desired allocation. The improved heuristic can perform better with an adaptive step size. Now we propose to improve the simple heuristic in a different way. Specifically, we develop a binary search to find the optimal workload allocation point. If $t_{c}<t_{g}$, we should allocate more work to the CPU side. Therefore, for the next step, we assign $r=(1+r) / 2$. If $t_{c}>t_{g}$, we should allocate more work to the GPU side. Therefore, we assign $r=r / 2$ for the next step. In order to guarantee the convergence. We define the precision threshold (i.e., $P_{t}$ ). $P_{t}$ is defined as: if the workload allocation in consecutive iterations is smaller than this value, the dynamic workload allocation terminates. By using such a binary search, we guarantee that our search can complete within $\log _{2}\left(100 / P_{t}\right)$ steps. The larger $P_{t}$ is, the faster the algorithm converges.

Improved Binary Search. In the design of Binary Search, we simply adjust the workload allocation in the unit of $r / 2$, where $r$ is the percentage of work allocated to CPU, by checking if $t_{c}$ or $t_{g}$ is greater. This can work well when the initial 


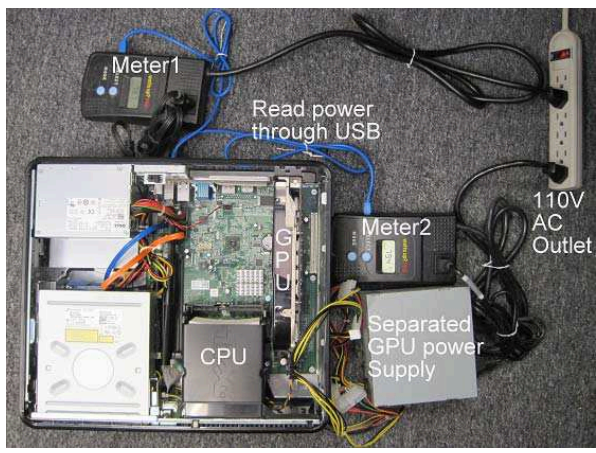

Figure 4: Hardware testbed used in our experiments, which includes a Dell Optiplex 580 desktop with an Nvidia GeForce GPU and an AMD Phenom II CPU, two power meters, one separated ATX power supply to power the GPU card. Meter1 measures the power of the CPU side, while Meter2 measures the power of the GPU side.

Table 3: Physical testbeds.

\begin{tabular}{|l|l|l|}
\hline & Testbed_A & Testbed_B \\
\hline$C P U$ & AMD Phenom II & Intel Xeon E3-1200 \\
\hline$G P U$ & GTX8800 & Tesla2070 \\
\hline$O S$ & Ubuntu 10.04 & Ubuntu 12.04 \\
\hline CUDA & 4.0 & 4.2 .18 \\
\hline
\end{tabular}

allocation is far from the optimal point. However, when the initial point is close to the optimal point, binary search may result in undesired oscillation around the optimal point by always adjusting $r / 2$. A key observation is that we may take the quantitative difference between $t_{c}$ and $t_{g}$ into consideration and then adjust the allocation proportionally based on the difference. Specifically, we calculate the new allocation $r^{\prime}$ using the following equation:

$$
\frac{t_{c}}{t_{g}}=\frac{r /(1-r)}{r^{\prime} /\left(1-r^{\prime}\right)}
$$

The key advantage of Improved Binary Search over the original Binary Search is that the workload is allocated to CPU and GPU proportionally based on their execution time difference. As a result, when the allocation is near the optimal point, we can avoid undesired oscillation caused by possible large workload change $(r / 2)$ in the original Binary Search. As shown in our experiment results in Section 7, Improved Binary Search does work the best among all the four proposed algorithms.

\section{Implementation}

In this section, we introduce our hardware testbeds and the implementation details of GreenGPU.

Due to the hardware configuration diversity of current high performance computer systems, we build two hardware testbeds (Testbed_A and Testbed_B in Table 3) to evaluate our proposed solution. Our primary testbed (i.e., Testbed_A) uses GeForce8800 GTX GPU [25]. GTX8800 has 16 Stream MultiProcessors (SM) with the $90 \mathrm{~nm}$ technology. By using the utility bandwidthTest from the Nvidia SDK, we derive the CPU to GPU bandwidth is $656.3 \mathrm{MB} / \mathrm{s}$ and the GPU to CPU bandwidth is $803.3 \mathrm{MB} / \mathrm{s}$. We use an off-the-shelf GPU card but not the latest card (e.g., Tesla series) because it is fully compatible with the management tools such nvidia-smi and nvidia-settings, which are required in our experiments. We select six frequency levels with equal distance in the dynamic range of the core part and memory part, respectively (e.g., $900 \mathrm{MHz}, 820 \mathrm{MHz}, 740 \mathrm{MHz}, 660 \mathrm{MHz}, 580 \mathrm{MHz}$, and $500 \mathrm{MHz}$ for GPU memory). Finer frequency levels may introduce large convergence time; coarser frequency levels may introduce large oscillation. Our selection is a trade-off between the convergence time and oscillation. For example, we use $576 \mathrm{MHz}$, $513 \mathrm{MHz}, 466 \mathrm{MHz}, 411 \mathrm{MHz}, 356 \mathrm{MHz}$, and $297 \mathrm{MHz}$ for GPU core frequency levels, and $900 \mathrm{MHz}, 820 \mathrm{MHz}, 740 \mathrm{MHz}, 660 \mathrm{MHz}$, $580 \mathrm{MHz}$, and $500 \mathrm{MHz}$ for GPU memory frequency levels. We enable Coolbits attribute of NVIDIA graphic card and use nvidia-settings shipped with the NVIDIA GPU driver to adjust the cores and memory frequencies of GPU. nvidia-smi [26] in Nvidia's toolkit is used to read the current GPU core and memory utilizations. The CPU in our physical testbed is a dual core AMD Phenom II X2 processor with four available frequency levels as $2.8 \mathrm{GHz}, 2.1 \mathrm{GHz}, 1.3 \mathrm{GHz}$, and $800 \mathrm{MHz}$. We use 2 Wattsup Pro power meters [33] to get the power readings. As shown in Figure 4, to measure the power consumption of the CPU and other parts of the system, we put the first power meter between the box and the $110 \mathrm{~V}$ AC wall outlet. Therefore, the first power meter measures the total power of the CPU side, including the motherboard, disk, and main memory. The GPU card is powered by an independent ATX power supply and its power consumption is measured with the second power meter placed between this ATX power supply and the wall outlet. The second power meter measures the total power of the GPU card.

We also build a secondary testbed (i.e., Testbed_B) to evaluate GreenGPU with the latest generation of GPU and CPU. However, due to support issues, nvidia-settings and the management library from Nvidia cannot change the frequency of Tesla2070. Therefore, on our secondary testbed, we focus on workload allocation algorithm evaluation. Specifically, we compare our simple heuristic and the binary search on Testbed_B. We use one Wattsup Pro power meter to measure the power consumption of the entire desktop and report the power reading.

We use Rodinia [2] and Nvidia SDK [27] as our workloads. Rodinia suite provides both CUDA and OpenMP implementations for their applications. We enlarge the data size and/or number of iterations of GPU computation kernels in order to get stable power reading (e.g, appending the input file content multiple times to increase the input file size). Table 2 shows the key parameters. Our workload selection covers all core and memory utilization characteristics and we also include workloads with dramatic fluctuation in terms of utilization (i.e., $Q G$ and $S C$ ) to test our GreenGPU framework. We 
Table 2: Summary of workloads used in our hardware experiments.

\begin{tabular}{|l|l|l|}
\hline Workloads & Enlargement & Description \\
\hline$b f s$ & 65536 iterations & High core and memory utilization \\
\hline$l u d$ & 10 iterations; 8192 by 8192 matrix & Medium core utilization, low memory utilization \\
\hline$n b o d y$ & 50 of iterations & High core and memory utilization \\
\hline$P F$ & 2048 by 2048 dimensions & Low core and memory utilization \\
\hline$Q G$ & 600 iterations; 16777216 points & Utilizations highly fluctuate \\
\hline srad_v2 & 2048 columns by 2048 rows & High core utilization, medium memory utilization \\
\hline hotspot & 2048 by 2048 grids of 600 iterations & Medium core utilization, low memory utilization \\
\hline kmeans & 988040 data points & Medium core utilization, low memory utilization \\
\hline streamcluster & 65536 points with 512 dimensions & Utilizations highly fluctuate \\
\hline
\end{tabular}

identify $Q G$ and $S C$ as high fluctuation workloads by studying the utilization traces of our workloads. We implement our frequency scaling part as a Python script and run the script as a background daemon process to adjust the GPU cores and memory frequency levels.

The workloads for two-tier design experiments are from Rodinia. Please note that although Open Computing Language (OpenCL) [16] is a C-based programming framework with promising heterogeneous processing support, the workload distribution between the CPU and GPU still requires lowlevel programming and memory management (e.g., programming a combination of OpenMP or pthreads with CUDA [27]). Therefore, we adopt a preliminary implementation structure as introduced in $[30,21]$ : multiple pthreads are launched in the main function. Some pthreads are in charge of CUDA execution (one pthread for one GPU), the other pthreads are deployed on the cores of the main CPU (one pthread for one core). We wrap the CPU and GPU implementations into different kernel functions and launch those kernels in different pthreads. We also implement parameters in those kernels such that the data size mapped to each kernel can be changed when it is invoked. We repeatedly call kernel functions with different data sizes to implement the workload division. We implement our workload division algorithm within the application code. Currently, the main program spawns pthreads for both CPU and GPU versions of implementation for the benchmarks in our evaluation. However, new programming interface like OpenCL offers opportunities to have just one copy of implementation that can be deployed on both CPU and GPU (or even DSP) platforms, which will significantly reduce the programming effort. Please note that the time and energy overheads of moving data between CPU and GPU have been included in our reported results.

Due to the future system integration trend, the energy and power management algorithm might need to be implemented on-chip [14]. However, since workload division within an application is a software problem by nature, it does not suit for on-chip hardware implementation. Therefore, we only sketch the possible hardware implementation for our frequency scaling tier. For monitoring, all the statistics we need in our algorithm can be derived from performance counters, which are already available on most modern CPUs and GPUs. There is no extra monitoring hardware required. The key part in the frequency scaling tier is the core-memory pair weight table. We need a $N \times M$ table to record the core-memory frequency pairs. Because the loss factor value is between 0 and 1. 8-bit precision is accurate enough for the purpose of picking up the largest weight. For our testbed with 6 core frequency levels and 6 memory levels, we only need a 36 bytes table $(6 \times 6 \times 8)$. The weights are updated based on Section 5.1. Please note the multipliers in Equations 1 to 3 are one coefficient fixed, they can be highly optimized to a simple shift-add logic. Scaled to 8 -bit and current $65 \mathrm{~nm}$ technology, the adder presented in [23] only consumes $0.001 \mathrm{~mm}^{2}$ and $12.5 \times 10^{-9} \mathrm{~J}$ each invocation. The leakage power of the 36-byte storage and the adder is negligible compared to that of billions of transistors in a modern GPU. Therefore, the hardware structure of our frequency scaling algorithm is area and energy efficient to be implemented on-chip.

\section{Experiments}

In this section, we first evaluate the GPU frequency scaling. We then test the workload division tier. Finally, we present the results of GreenGPU as a holistic energy management solution.

\subsection{Frequency Scaling for GPU Cores and Memory}

In this experiment, we enable the frequency scaling tier but disable the workload division tier (i.e., all the workloads are put to the GPU) to test the performance and energy savings of the frequency scaling algorithm. We use the best-performance policy as our baseline. Best-performance sets both core and memory frequencies always at the highest level (i.e., $576 \mathrm{MHz}$ for cores and $900 \mathrm{MHz}$ for memory). We compare our frequency scaling algorithm with best-performance to show that our algorithm can achieve considerable energy savings with only negligible performance loss.

Figure 5 shows the trace file of a typical run of our frequency scaling with the streamcluster workload. Our experiment starts with the frequencies of cores and memory running at the lowest levels, which is the default case for a GPU. Figures $5 \mathrm{a}$ and $5 \mathrm{~b}$ show that the core and memory frequencies are generally directed by their utilizations. In Figure 5a, 


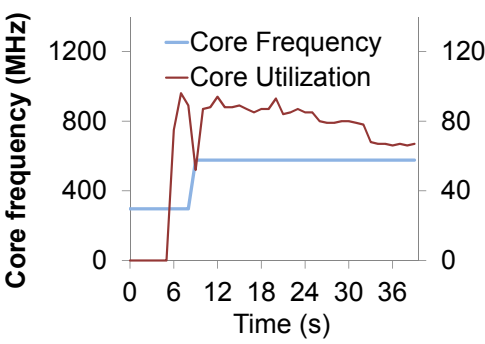

(a) Core part trace

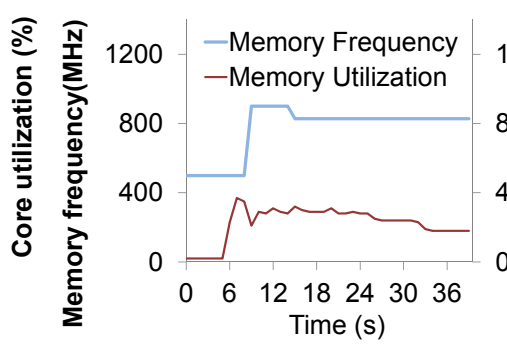

(b) Memory part trace

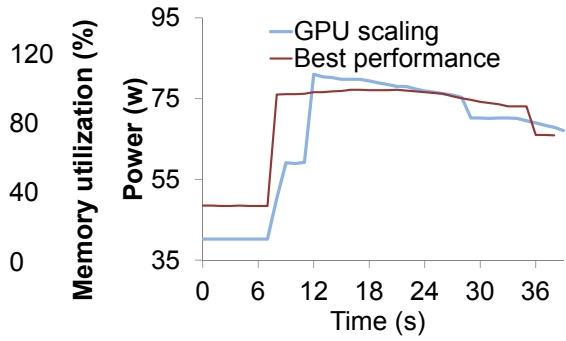

(c) Power trace

Figure 5: Frequency scaling algorithm adjusts the frequencies of GPU cores and memory based on their utilizations respectively to save energy without increasing execution time.

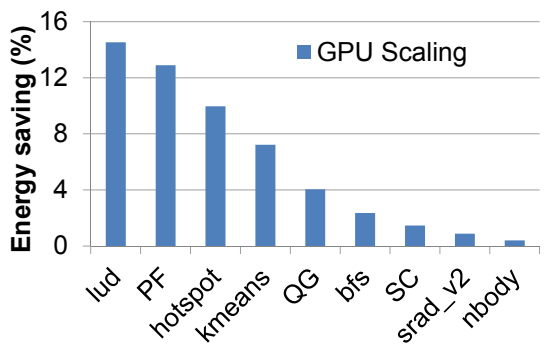

(a) GPU energy

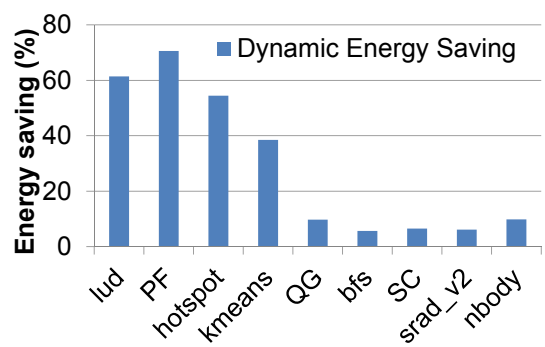

(b) GPU dynamic energy

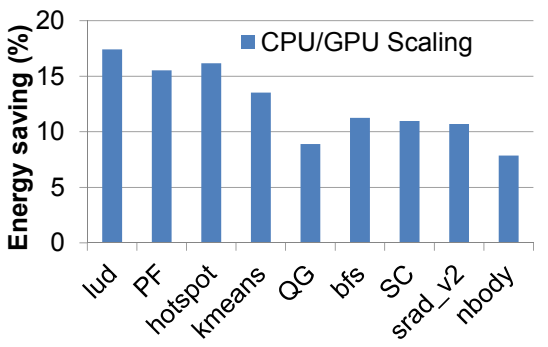

(c) System energy

Figure 6: Energy saving compared with best-performance for different workloads.

the utilization of cores starts to ramp up from the 6th second. Since our frequency scaling interval is $3 \mathrm{~s}$ in this test, at the 9th second (i.e., the immediate next period after the utilization increase), the frequency of cores is adjusted to be higher. Since our algorithm evaluates the loss value of all possible frequency levels, it can adjust the GPU core and memory frequencies directly to the best levels according to the utilizations. In Figure $5 \mathrm{~b}$, the memory frequency converges to $820 \mathrm{MHz}$, which is lower than the peak frequency (i.e., $900 \mathrm{Mhz}$ ) and so results in energy savings. As shown in Figure 5c, the average power consumption of our algorithm is lower than that of best-performance throughout the experiment, but the execution time (i.e., performance) is similar. As a result, the energy efficiency is improved.

Figure 6 presents the energy saving percentage of our scheme compared with best-performance for different workloads. In Figure 6a, GPU scaling is the measured result of our corememory coordinated frequency scaling algorithm. Our algorithm saves $5.97 \%$ on average and up to $14.53 \%$ of GPU energy. In Figure 6b, we present the energy savings in terms of dynamic GPU energy. Dynamic Energy Saving numbers are calcuated by subtracting the idle energy from the runtime energy. Figure $6 \mathrm{~b}$ shows that our approach saves $29.2 \%$ of dynamic energy on average with only $2.95 \%$ longer execution time than best-performance. In Figure 6c, CPU/GPU scaling is the result when we throttle both the CPU and GPU for maximized energy savings. The key idea is that the CPU frequency can be throttled down for energy savings with asynchronized GPU-CPU communications, when the GPU part is doing all the computation. However, due to the limitations in the implementation of synchronized GPU-CPU communications used in our benchmarks, the CPU has a utilization of $100 \%$ even when it is idling and the GPU is doing all the work. As a result, the on-demand CPU frequency governor of Linux used in GreenGPU fails to throttle the CPU frequency for energy savings. We therefore emulate this case to highlight the energy saving potential of dynamically throttling both the CPU and GPU. In our emulation, we conservatively assume that the CPU frequency cannot be throttled if the CPU needs to communicate with the GPU at any time, such as the workload launching and ending times. When the CPU is idling and its frequency can be throttled without impacting the system performance, we replace the CPU energy with the average CPU energy at the lowest frequency level to emulate that CPU frequency is throttled to the lowest level. Figure $6 \mathrm{c}$ shows that the average energy saving is $12.48 \%$ if both CPU and GPU are throttled. Note that we do emulation only in Figure 6c.

Based on Figure 6 and the corresponding workloads in Table 2, we can make the following observations. First, for workloads with phase fluctuation, such as $Q G$ and streamcluster, our scheme can achieve energy savings because we dynamically detect the on-line utilization information of the cores and memory and dynamically adjust frequencies accordingly. Second, for applications with a lower average utiliza- 
tion (either core part or memory part, such as $P F$ and $l u d$ ), our scheme yields good energy savings. However, for the applications with high utilization rates, such as $b f s$, the energy savings are smaller. This is because if all the resources are occupied, throttling either core or memory frequency will significantly increase execution time, resulting in increased energy consumption.

To summarize this part, our scheme is effective for both phase-stable and phase-fluctuating workloads, and it performs better for the workloads with low utilizations of either GPU cores or memory than the workloads with high utilizations of both GPU cores and memory.

\subsection{Workload Division between GPU and CPU}

In this section, we enable the workload division tier but disable the frequency scaling tier to investigate the effectiveness of dynamic workload division. Note that we use the simple heuristic algorithm designed in Section 5.2. The other three algorithms can lead to even better results and are comparatively evaluated in Section 7.4.

Figure 7 presents the traces of the workload division for kmeans and hotspot. In Figure 7, the $\mathrm{X}$-axis is the iteration sequence number; the left Y-axis is the workload division percentage; the right $\mathrm{Y}$-axis is the execution time. The triangle dot is the execution time of the GPU part in the corresponding iteration. The round dot is the execution time of the CPU part in the corresponding iteration. In Figure 7a, the initial division ratio is set to be $30 \%$ workloads on the CPU part. We pick up $30 \%$ here in order to have a faster convergence. In real usage, this value can be set to an arbitrary ratio (e.g., $50 \%$ ). Since we use 5\% as workload division step, in the worst case, we need 10 iterations if we start from the 50\% division point. In our experiments, we find that setting initial ratio to $50 \%$ to $30 \%$ can help to converge the balanced workload division in a shorter time. However, we will show that our algorithm converges to the balanced workload division regardless of this initial division ratio. In the 1st iteration, the CPU execution time is much longer than the GPU execution time. Our division algorithm takes one piece of workload from the CPU and assigns it to the GPU part. The execution time difference between the CPU and GPU becomes smaller in the 2nd iteration. But the CPU execution time is still much longer than the GPU execution time. Our division algorithm takes one more piece of workload from the CPU and assigns it to the GPU part. In the 3rd iteration, the execution times of the two parts become even closer. The process repeats until the execution times on both sides are roughly the same after 4 iterations. The rationale of this adjustment is to minimize the idling energy caused by waiting for the slower side, as discussed in Section 1. Figure $7 \mathrm{~b}$ shows a similar case for another workload, hotspot. As demonstrated in figures $7 \mathrm{a}$ and $7 \mathrm{~b}$, our algorithm can dynamically adjust the workload division based on the runtime execution time difference between the CPU and GPU parts in a
GPU-CPU system regardless of its initial division ratio. To examine how close the result of our workload division algorithm is to the optimal division point with the minimum energy consumption, we have also conducted a series of experiments to test static workload division from $0 / 100$ to $100 / 0$ (CPU/GPU) with a step size of 5 . For kmeans, we find that the energyminimum division is $15 / 85$ (CPU/GPU). In comparison, our algorithm converges to 20/80. For hotspot, the energy minimum division is 50/50 (CPU/GPU), while our algorithm converges exactly to 50/50 and obtains $99 \%$ of the maximum saving. The $1 \%$ difference is mainly due to 1 ) the higher energy consumption before the convergence, and 2) the overheads of dynamic workload division. For the workload division part only, our solution only has $5.45 \%$ longer execution time than the optimal division.

\subsection{GreenGPU as a Holistic Solution}

We now enable both the workload division and the frequency scaling tiers to test GreenGPU as a holistic solution. We show that such a holistic solution leads to more energy savings than each individual scheme.

Figure 8 shows the runtime traces of hotspot and kmeans. In Figure 8, the $\mathrm{X}$-axis is the iteration sequence number; the left Y-axis is the workload division percentage; the right Yaxis is the energy consumption in each iteration. The triangle dots, hollow round dots, squares are the energy consumption of GreenGPU, Division (with frequency scaling disabled), and Frequency-scaling (with workload division disabled) in the corresponding iteration. In Figure 8a, GreenGPU consumes less energy than Division and Frequency-scaling. Compared with Division, GreenGPU's frequency scaling units observe the energy saving opportunities when the GPU cores, GPU memory, or CPU has a low utilization. GreenGPU then throttles the frequencies of them based on the proposed frequency scaling algorithm. By doing that, GreenGPU achieves lower energy consumption than Division. Compared with Frequencyscaling, GreenGPU has more energy savings by dynamically dividing workloads between the GPU and CPU. By balancing the workload between GPU and CPU, GreenGPU can minimize the time idle energy consumed by GPU or CPU to wait for the other side to finish. For hotspot, GreenGPU achieves $7.88 \%$ more energy saving than Division and $28.76 \%$ more than Frequency-scaling. Figure $8 \mathrm{~b}$ shows a similar case for a different workload, kmeans. In our testbed, Division contribute more to energy saving than Frequency-scaling in holistic solution because nvidia-settings on GeForce 8800 only conducts frequency scaling [26]. If DVFS is enabled, we expect more energy saving can be achieved from frequency scaling. GreenGPU saves 1.6\% more than Division and 12.05\% more than frequency scaling for kmeans. The default runtime configuration of Rodinia is that all the workloads are allocated to the GPU and all the frequencies are at their peak levels [2]. Compared with that, GreenGPU can achieve on average 


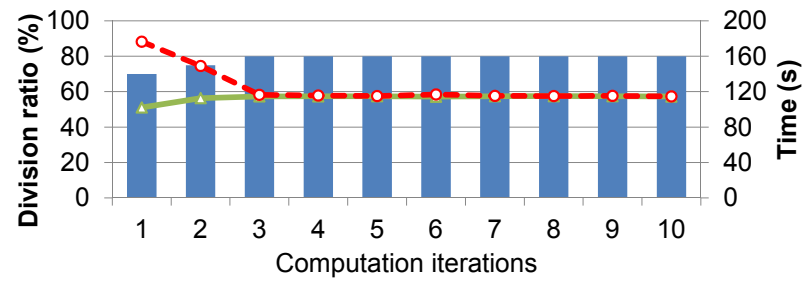

- GPU

(a) Workload division and execution time of kmeans.

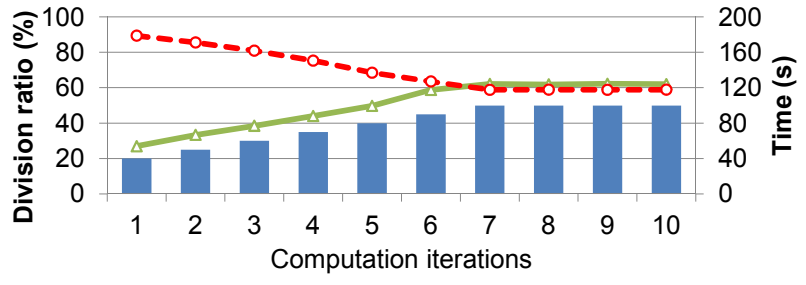

- GPU WM: CPU $\triangle-$ GPU Execution Time $-\infty$ CPU Execution Time

(b) Workload division and execution time of hotspot.

Figure 7: Workload division algorithm adjusts the workload allocation between the CPU and GPU parts to minimize idling energy on either side caused by waiting for the other (slower) side.

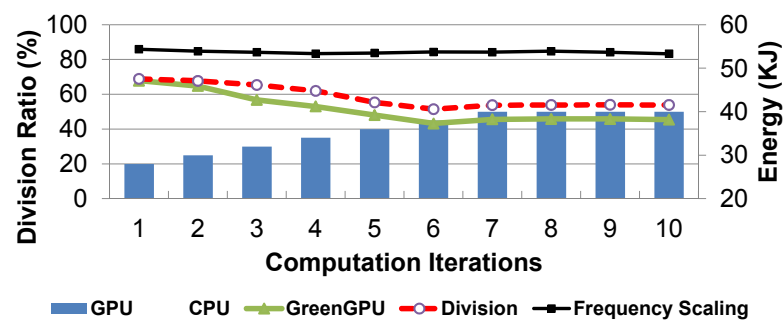

(a) hotspot.

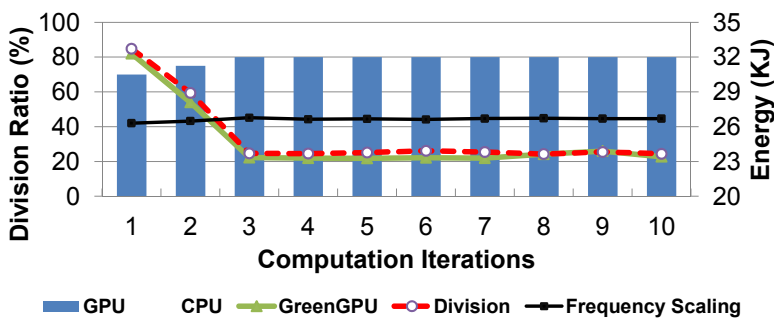

(b) kmeans.

Figure 8: Energy and workload division ratio trace in respect of the iterations. GreenGPU outperforms workload division only and frequency scaling only on energy savings.

$21.04 \%$ energy saving for kmeans and hotspot. For the holistic solution as a whole, GreenGPU has $1.7 \%$ longer execution time than workload-division-only.

\subsection{Workload Division Algorithm Comparison}

In this section, we focus on the workload allocation algorithm comparison between the four algorithms designed in 5.2: The simple heuristic, the improved heuristic with adaptive step size, the binary search algorithm, and the improved binary search. While all the experiments in the previous sections are conducted on our Testbed_A, the tests in this section are conducted on our Testbed_B. The reasons that we build two hardware testbeds are: a) we want to evaluate our proposed algorithm on different hardware and software configurations; b) the latest Nvidia Tesla GPU does not respond to the frequency scaling option in nvidia-setting tool. We thus cannot manually set the frequency of Tesla GPU. Therefore, we only use Testbed_B to evaluate the four workload allocation algorithms.

In this section, we mainly present the results of the workload hotspot. The results of other workloads are similar and briefly discussed at the end of the section due to space limitations. In Figure 9, we present the runtime trace of the Simple Heuristic workload allocation algorithm with different initial points. The allocation step is $3 \%$ of the workload (i.e., Hotspot). We can see that, when the execution time on GPU is longer than that of CPU, the algorithm shifts more workload to CPU; when the execution time on CPU is longer than that of
GPU, the algorithm allocates more workload to GPU. Therefore, even with different initial points, such a simple heuristic can direct the entire system converge to the optimal CPU/GPU allocation point (e.g., CPU takes approximately $80 \%$ of the workload). However, the initial point impacts the number of the iterations to converge. In general, if the initial point is close to the converge point (i.e., the $90 \%$ initial point case), the heuristic algorithm takes fewer iterations to converge. If the initial point is far from the converge point (e.g., the $50 \%$ and $15 \%$ initial points), the heuristic algorithm takes more iterations to converge.

The results of running the Improved Heuristic workload allocation algorithm with different initial points are shown in Figure 10. The initial allocation adjustment step size is $15 \%$ for the workload (i.e., Hotspot) and the threshold for stopping adjustment is $1 \%$. We can see that Improved Heuristic converges much faster than Simple Heuristic at beginning of the trace, due to its initial large step size. For example, when the initial allocation is CPU taking 15\%, Improved Heuristic takes only 5 steps to start having oscillation, while Simple Heuristic takes 22 steps. After the first oscillation in iteration 6, Improved Heuristic halves the step size to $8 \%$, which leads to another (smaller) oscillation. Improved Heuristic then continue to halve the step size to $4 \%$ and then $2 \%$ and $1 \%$. In addition to its faster convergence, Improved Heuristic halves can converge to the optimal point with a better accuracy than Simple Heuristic due to its smaller final step (i.e., 1\%), while 

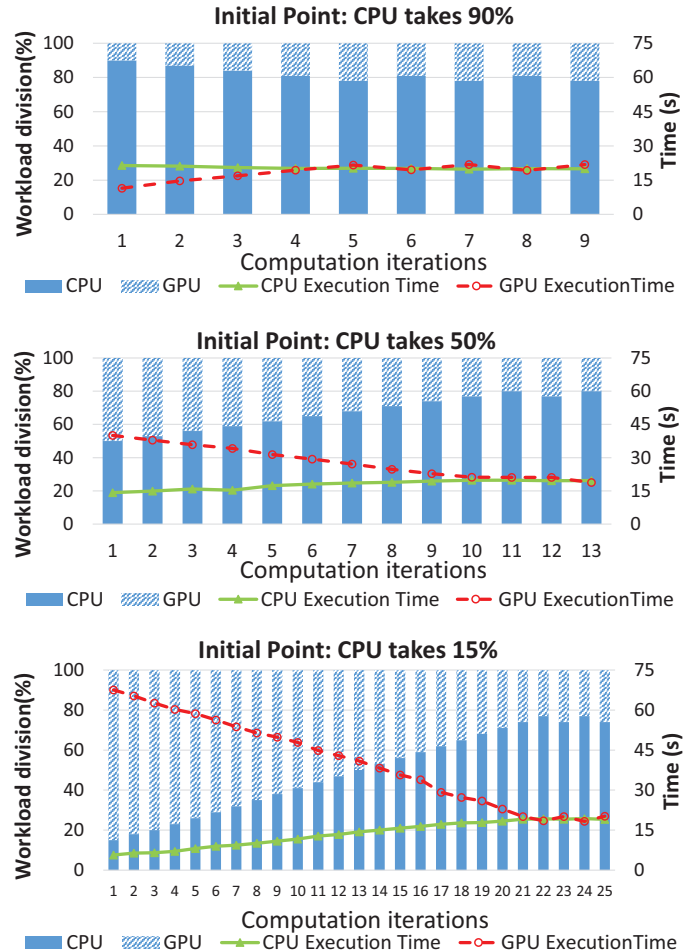

Figure 9: Simple Heuristic workload allocation on Hotspot workload with a fixed step size of 3\%. Despite different initial points, the heuristic converges to the optimal allocation point (e.g., CPU takes approximately $80 \%$ of the workload). However, the initial point impacts the number of iterations to converge.
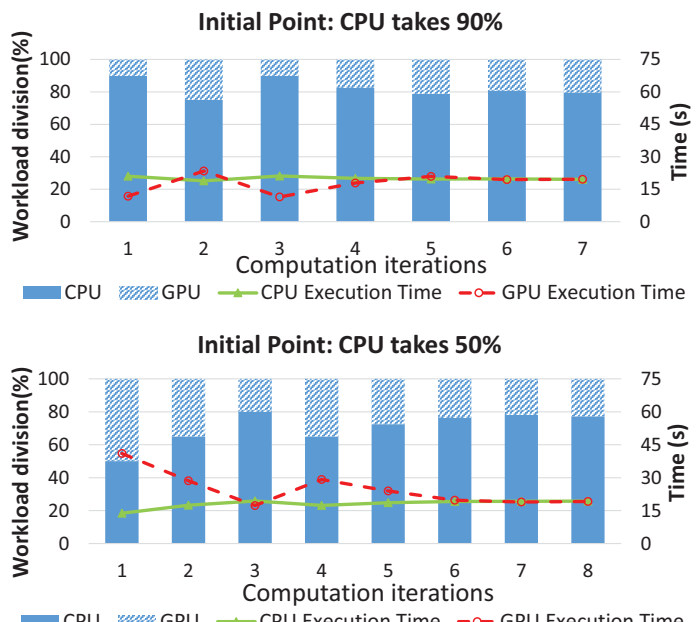

$-\mathrm{CPU}$ mss GPU - CPU Execution Time -o- GPU Execution Time

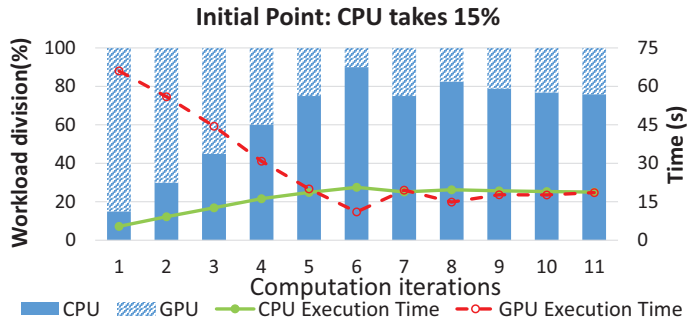

Figure 10: Improved Heuristic on Hotspot workload with an initial step size of $15 \%$ and different initial points. Improved Heuristic converges much faster than Simple Heuristic by dynamically adapting the step size. Moreover, the accuracy of approaching the optimal point can be further improved by having a smaller final step size (1\%).

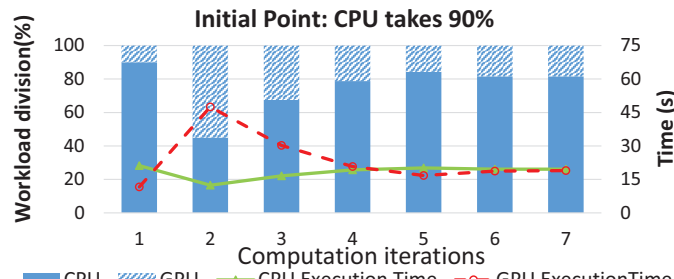

CPU ma GPU $=$ CPU Execution Time -o- GPU ExecutionTime

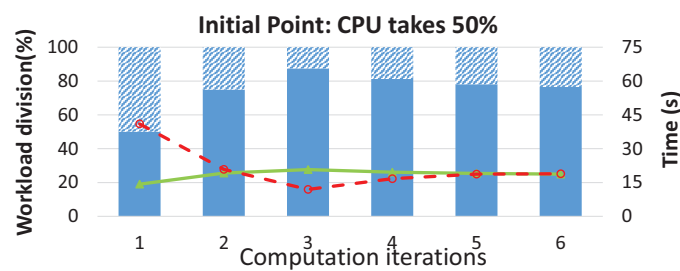

- CPU

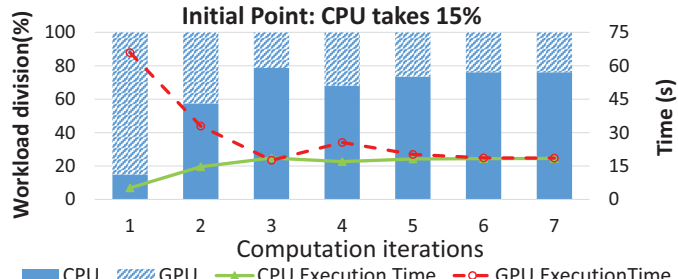

Figure 11: Binary Search workload allocation on Hotspot workload with different initial points. The key benefit of Binary Search is that it does rely on any step size, because it dynamically allocates the half workload of the slower one (CPU or GPU) to the faster one. Moreover, all the test cases converge with in $\log _{2}(100)=7$ iterations.
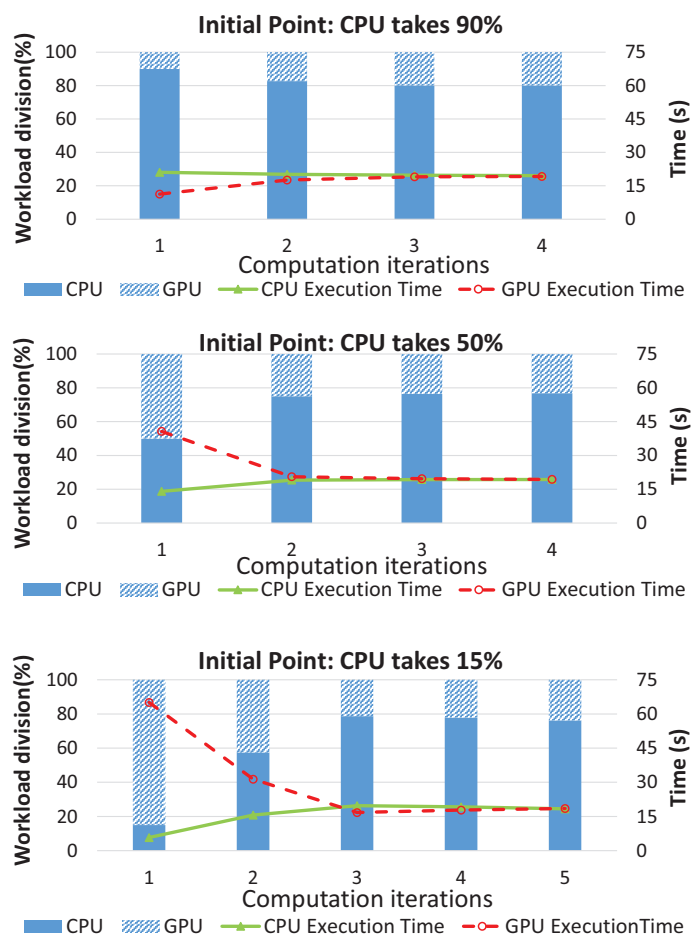

Figure 12: Improved Binary search workload allocation on Hotspot workload with different initial points. Improved Binary further improves Binary Search by quantitatively considering the execution time difference between CPU and GPU and proportionally adjusting the workload allocation based on the difference. As a result, the efficiency of workload allocation is significantly improved. 
Simple Heuristic adopts a fixed step size of $3 \%$. The accuracy is $19.75 \%$ for Improved Heuristic and either $19 \%$ or $22 \%$ for Simple Heuristic. Moreover, the accuracy of approaching the optimal point can be further improved by having an even smaller final step size at the cost of a longer convergence time. A larger initial step size (e.g., 20\%) or a faster rate (e.g., dividing by 3 instead of halving) can also be used if a shorter convergence time is desired.

Figure 11 shows the result of running the Binary Search workload allocation algorithm with different initial points. We set $P_{t}=1 \%$ (i.e., $P_{t}$ is the precision threshold, defined in Section 5.2). In general, we can see that binary search also directs the workload allocation to converge to the optimal point (e.g., CPU takes $80 \%$ workload). In addition, the initial point setting does not impact the number of convergence iterations a lot. In all the cases, the binary search converges within $\log _{2}(100)=7$ iterations. However, when the initial point is near the optimal allocation point (e.g., initial point is $90 \%$ ), large oscillation occurs around the optimal, which can reduce the efficiency of this algorithm. Also, as shown in the case with an initial point of $90 \%$, Binary Search unnecessarily allocates too much workload to GPU in the second iteration, despite that the execution times of CPU and GPU are close to each other in the first iteration. This is because Binary Search does not consider the difference between CPU and GPU execution times and so cannot adjust the allocation proportionally based on the difference, which motivates the design of Improved Binary Search.

We now examine the results of Improved Binary Search, which are shown in Figure 12. We test the same initial allocation points: $15 \%, 50 \%$, and $90 \%$. We set $P_{t}=1 \%$ for a fair comparison with Binary Search. We can see from the same case with an initial point of $10 \%$ that Improved Binary Search avoids having the large oscillation that Binary Search causes, by allocating workload proportionally based on the execution time difference. As a result, the efficiency of workload allocation is significantly improved, resulting in a much shorter convergence time. For example, only 4 iterations are needed for an initial point of $90 \%$. Even when the initial point is far away from the optimal point (e.g., 15\%), Improved Binary Search converges within only 5 iterations.

We now briefly discuss the results of running different workloads (i.e., Kmeans), as those results are similar to those of hotspot and so not presented completely due to space limitations. Figure 13 shows the trace of running the Binary Search workload allocation algorithm. We can see that Binary Search also directs Kmeans workload allocation to converge. In addition, the initial point setting does not significantly impact the number of convergence iterations. In all the cases, Binary Search converges within $\log _{2}(100)=7$ iterations.

Figure 14 shows the results of running Hotspot workload in different test cases and compares the number of iterations for the four allocation algorithms to converge. H $3 \%$ means that we use the Simple Heuristic allocation with $3 \%$ step size.
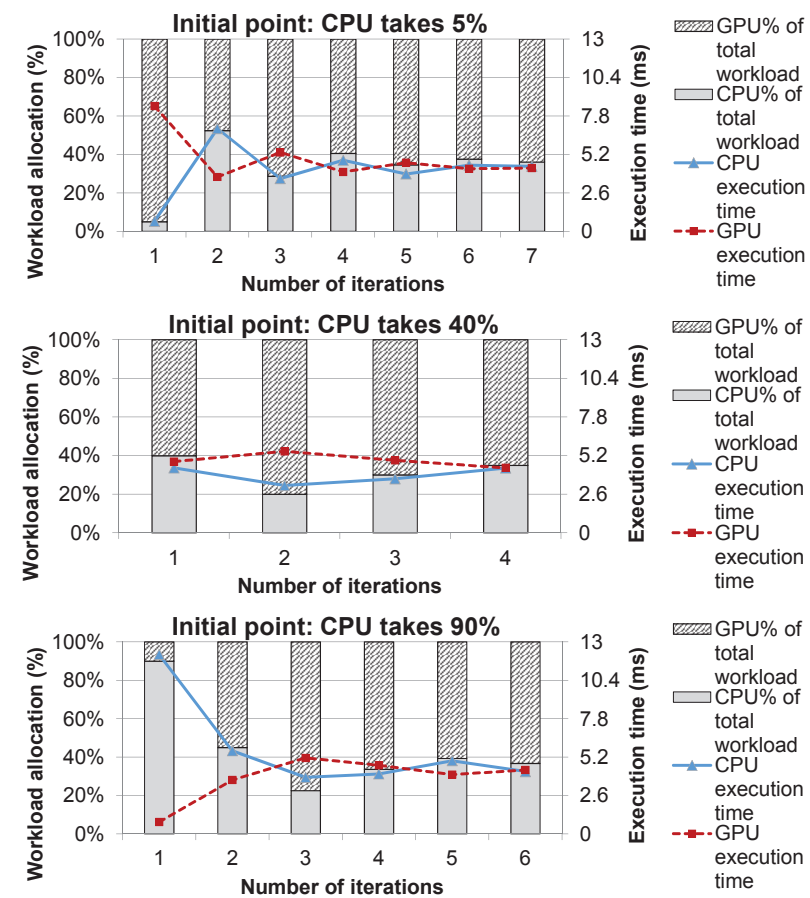

Figure 13: Binary Search workload allocation on Kmeans workload with different initial points. Despite different workloads, Binary Search converges within $\log _{2}(100)$ times of iterations.

The larger the step size is, the less time it takes to converge. IH $10 \%$ means that we use the Improved Heuristic allocation with $10 \%$ as initial step size. B $1 \%$ means that we use the Binary Search allocation with $1 \% P_{t}$. By using such a binary search, we guarantee that the search can complete within $\log _{2}\left(100 / P_{t}\right)$ times. The larger $P_{t}$ is, the faster the algorithm converges. IB $1 \%$ also means that we use threshold $P_{t}=1 \%$. We have also tested the Kmeans workload. On average, Improve Binary results in the smallest number of iterations for convergence. Figure 15 presents only the results of Simple Heuristic and Binary Search due to space limitations. As can seen clearly, the results of Kmeans are similar to those of Hotspot, except that the optimal allocation point is different (approximately 40\%).

In the comparison of the four workload allocation algorithms, Simple Heuristic is most intuitive and adjusts the allocation dynamically with a fixed step size. However, such a fixed step leads to a long convergence time when the initial allocation is far from the optimal point and a poor accuracy. As a result, we have proposed two ways to dynamically adapt the step size: Improved Heuristic and Binary Search. Improved Heuristic, if configured well with a large initial step size and a fast rate, can converge quickly to the optimal point. Binary Search approaches the problem in a different way and so does not depend on the selection of initial step size. Improved Binary Search further improves Binary Search by taking into account the the difference between CPU and GPU execution 


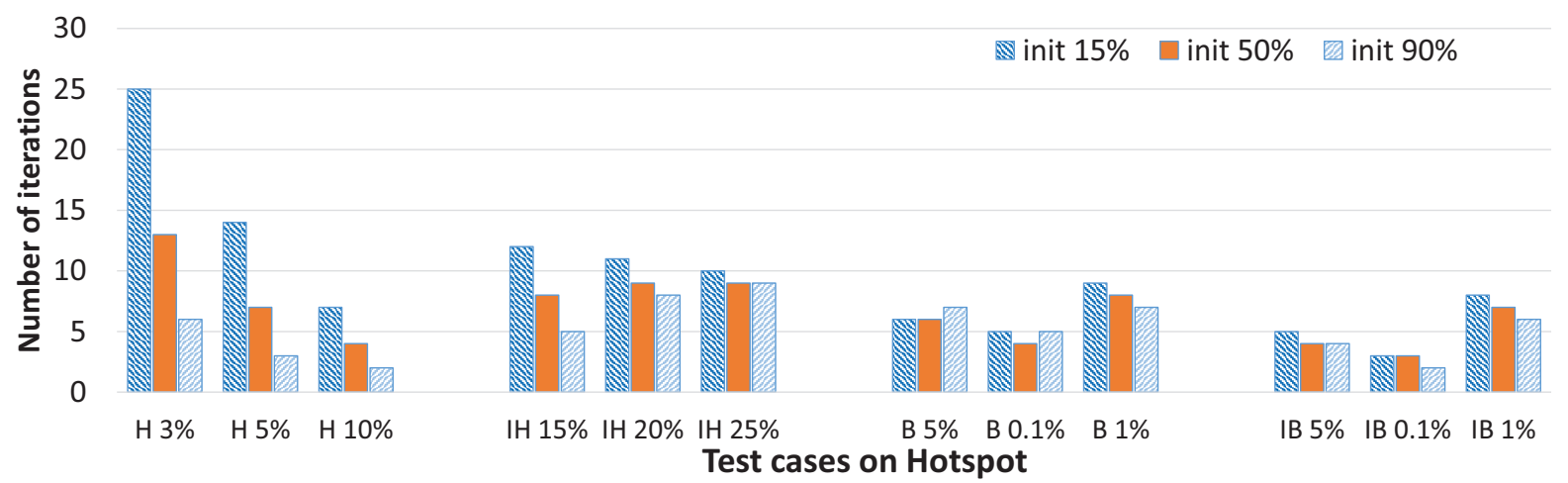

Figure 14: The number of iterations to converge needed by the four allocation algorithms, for the Hotspot workload under different test cases and initial allocation points. H 3\% means that we use Simple Heuristic with a 3\% step size. The larger the step size is, the less time it takes to converge. IH $10 \%$ means that we use Improved Heuristic with $10 \%$ as the initial step size. B $1 \%$ means that we use the Binary Search allocation with $1 \% P_{t}$. The larger $P_{t}$ is, the faster the algorithm converges. IB $1 \%$ also means that we use threshold $P_{t}=1 \%$ for Improve Binary. On average, Improve Binary results in the smallest number of iterations for convergence.

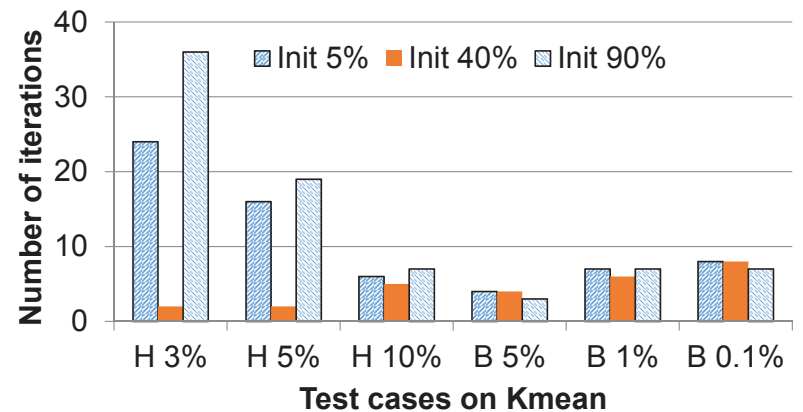

Figure 15: The number of iterations to converge needed by the four allocation algorithms for Kmeans. The results are similar to those of Hotspot. Due to space limitations, we present only the results of Simple Heuristic and Binary Search here.

times.

Figure 16 shows the total energy consumption of these four algorithms to finish the same workload (i.e., Hotspot). For Simple Heuristic, we use a fixed step size of 3\%. For Improved Heuristic, we use an initial step size of $15 \%$. In general, Improved Binary consumes the least amount of energy while Simple Heuristic costs the most to execute the same amount of workload. There are two major reasons: a) Due to its fastest convergence to the optimal allocation point, Improved Binary saves more energy than the other three algorithms because it avoids keeping either CPU or GPU idling, b) Improved Binary also avoids large oscillation when initial point is near the optimal point, which results in smaller allocation overheads. Also, for the Simple Heuristic and Improved Heuristic algorithms, the initial point significantly affects the total energy consumption. In general, the farther the initial point is from the optimal allocation point, the more energy the algorithm will consume, due to longer convergence to the

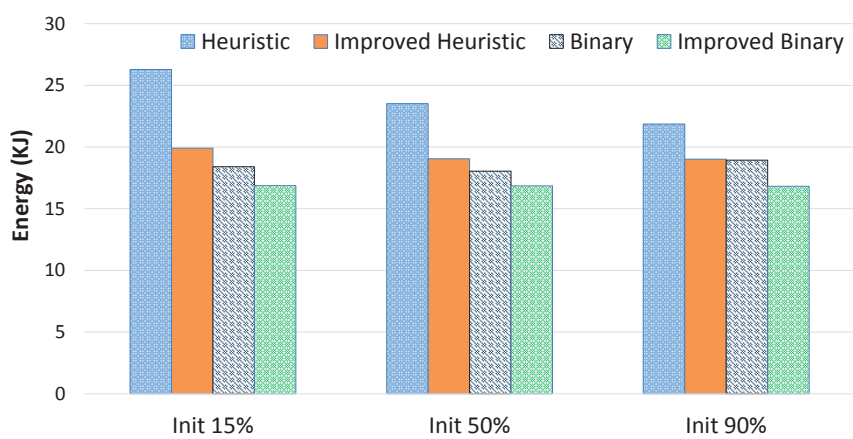

Figure 16: The overall energy comparison between the four workload allocation algorithms. Improved Binary saves more energy than the other three algorithms because: a) It avoids keeping either CPU or GPU idling. b) Improved Binary also avoids large oscillation when initial point is near the optimal point, which results in smaller allocation overheads.

optimal point. One exception is that Improved Heuristic consumes more energy when the initial step size is $25 \%$. This is because with a large initial step size, Improved Heuristic has unnecessary large oscillation when the initial allocation is already near the optimal point. In comparison to the two heuristic algorithms, Binary Search and Improved Binary are less sensitive to the initial point and do not need a careful selection of step size.

\section{Conclusion}

Current research on GPU-CPU architectures focuses mainly on the performance aspects, while the energy efficiency of such systems receives much less attention. There are few existing studies that start to lower the energy consumption of GPU-CPU architectures, but they address either GPU or CPU 
in an isolated manner and thus cannot achieve maximized energy savings. In this paper, we have presented GreenGPU, a holistic energy management framework for GPU-CPU heterogeneous architectures. Our solution features a two-tier design. In the first tier, GreenGPU dynamically splits and distributes workloads to GPU and CPU based on the workload characteristics, such that both sides can finish approximately at the same time. As a result, the energy wasted on staying idle and waiting for the slower side to finish is minimized. We comparatively discuss four dynamic workload allocation algorithms: a simple heuristic with fixed step size, an improved heuristic with adaptive step size, and two binary search-style algorithms. Our results show that Improved Binary leads to the smallest number of iterations for convergence and lowest energy consumption, due to its quantitative consideration of execution time differences between CPU and GPU. In the second tier, GreenGPU dynamically throttles the frequencies of GPU cores and memory in a coordinated manner, based on their utilizations, for maximized energy savings with only marginal performance degradation. Likewise, the frequency and voltage of the CPU are scaled similarly. We have implemented GreenGPU using the CUDA framework on two hardware testbeds. Experiment results show that GreenGPU achieves $21.04 \%$ average energy savings and outperform several well-designed baselines.

\section{References}

[1] Q. Cai, J. Gonzalez, R. Rakvic, G. Magklis, P. Chaparro, and A. Gonzalez, "Meeting points: using thread criticality to adapt multicore hardware to parallel regions," in PACT, 2008.

[2] S. Che, M. Boyer, J. Meng, D. Tarjan, J. Sheaffer, S.-H. Lee, and K. Skadron, "Rodinia: A benchmark suite for heterogeneous computing," in IISWC, 2009.

[3] S. Collange, D. Defour, and A. Tisserand, "Power consumption of gpus from a software perspective," Computational Science, pp. 914-923, 2009.

[4] J. Dean and S. Ghemawat, "Mapreduce: simplified data processing on large clusters," 2004.

[5] Q. Deng, D. Meisner, A. Bhattacharjee, T. F. Wenisch, and R. Bianchini, "Coscale: Coordinating cpu and memory system dvfs in server systems," in MICRO, 2012.

[6] G. Dhiman and T. S. Rosing, "Dynamic voltage frequency scaling for multi-tasking systems using online learning," in ISLPED, 2007.

[7] V. Freund and R. Schapire, "A decision-theoretic generalization of online learning and an application to boosting," JCSS, vol. 55, 1997.

[8] GREEN500.org, "The green500 list," http://www.green500.org/lists/ 2010/11/top/list.php, 2010.

[9] E. Grochowski and M. Annavaram, "Energy per instruction trends in intel microprocessors," Intel Microarchitecture Research Lab, Tech. Rep., 2006.

[10] S. Herbert and D. Marculescu, "Variation-aware dynamic voltage/frequency scaling," in HPCA, 2009.

[11] C. Hong, D. Chen, W. Chen, W. Zheng, and H. Lin, "MapCG: writing parallel program portable between CPU and GPU," in PACT, 2010.

[12] S. Hong and H. Kim, "An integrated GPU power and performance model," in ISCA, 2010.

[13] C.-H. Hsu and U. Kremer, "The design, implementation, and evaluation of a compiler algorithm for CPU energy reduction," in PLDI, 2003.

[14] Intel, "Intel turbo boost technology," http://www.intel.com/technology/ turboboost/, 2007.
[15] B. Jang, D. Schaa, P. Mistry, and D. Kaeli, "Exploiting memory access patterns to improve memory performance in data-parallel architectures," Parallel and Distributed Systems, IEEE Transactions on, vol. 22, no. 1, pp. 105-118, 2011.

[16] Khronos, "OpenCL - the open standard for parallel programming of heterogeneous systems," http://www.khronos.org/opencl, 2010.

[17] J. Lee, V. Sathisha, M. Schulte, K. Compton, and N. S. Kim, "Improving throughput of power-constrained gpus using dynamic voltage/frequency and core scaling," in PACT, 2011.

[18] J. Li, J. Martinez, and M. Huang, "The thrifty barrier: Energy-aware synchronization in shared-memory multiprocessors," in HPCA, 2004.

[19] N. Littlestone and M. Warmuth, "The weighted majority algorithm," Inf. Comput., vol. 108, pp. 212-261, 1994.

[20] C. Liu, A. Sivasubramaniam, M. Kandemir, and M. J. Irwin, "Exploiting barriers to optimize power consumption of CMPs," in IPDPS, 2005.

[21] C.-K. Luk, S. Hong, and H. Kim, "Qilin: exploiting parallelism on heterogeneous multiprocessors with adaptive mapping," in MICRO, 2009.

[22] K. Ma, X. Li, W. Chen, C. Zhang, and X. Wang, "Greengpu: A holistic approach to energy efficiency in gpu-cpu heterogeneous architectures," in Proceedings of the 41st International Conference on Parallel Processing (ICPP 2012), 2012.

[23] S. Mathew, M. Anders, R. Krishnamurthy, and S. Borkar, "A 4-GHz 130-nm address generation unit with 32-bit sparse-tree adder core," in ISSCC, 2003.

[24] T. N. Minh and L. Wolters, "Modeling parallel system workloads with temporal locality," in Job Scheduling Strategies for Parallel Processing. Springer-Verlag, 2009.

[25] NVIDIA, "Geforce 8800," http://www.nvidia.com/page/geforce_8800. html, 2010.

[26] — "Nvidia-smi," http://www.nvidia.com/, 2010

[27] — " "Cuda toolkit 3.2 downloads," http://developer.nvidia.com/ cuda-toolkit-32-downloads, 2011.

[28] OLCF, "Introducing Titan," http://http://www.olcf.ornl.gov/titan/.

[29] V. Pallipadi and A. Starikovskiy, "The ondemand governor," http://ftp. kernel.org/pub/linux/kernel/, 2006.

[30] V. T. Ravi, W. Ma, D. Chiu, and G. Agrawal, "Compiler and runtime support for enabling generalized reduction computations on heterogeneous parallel configurations," in ICS, 2010.

[31] K. Skadron, M. Stan, K. Sankaranarayanan, W. Huang, S. Velusamy, and D. Tarjan, "Temperature-aware microarchitecture: Modeling and implementation," ACM Trans. Archit. Code Optim., vol. 1, pp. 94-125, 2004.

[32] G. Wang and X. Ren, "Power-efficient work distribution method for CPU-GPU heterogeneous system," in ISPA, 2010.

[33] Wattsupmeters, "Watts up pro power meter," http://www.wattsupmeters. com, 2010.

[34] Q. Wu, P. Juang, M. Martonosi, and D. W. Clark, "Formal online methods for voltage/frequency control in multiple clock domain microprocessors," in ASPLOS, 2004. 